\title{
Fast and sharp decrease in calprotectin predicts remission by infliximab in anti-TNF naive patients with ulcerative colitis
}

\author{
M. De Vos ${ }^{a, *}$, O. Dewit ${ }^{b}$, G. D'Haens ${ }^{c}$, F. Baert $^{\text {d }}$, F. Fontaine $^{\text {e }}$, \\ S. Vermeire ${ }^{f}$, D. Franchimont ${ }^{g}$, T. Moreels $^{h}{ }^{\text {, D. Staessen }}{ }^{i}$, L. Terriere $^{j}$, \\ B. Vander Cruyssen ${ }^{a}$, E. Louis ${ }^{k}$ on behalf of BIRD
}

a Ghent University Hospital, Gent, Belgium

b Université Catholique Louvain St Luc, Brussel,Belgium

c Imelda Hospital, Bonheiden, Belgium and Academic Medical Centre, Amsterdam, The Netherlands

${ }^{\mathrm{d}}$ H.Hart Ziekenhuis; Roeselare-Menen, Belgium

e Clinique St Joseph, Liege, Belgium

f University Hospital Leuven, Belgium

${ }^{g}$ Université libre Bruxelles, Belgium

${ }^{\mathrm{h}}$ Antwerp University Hospital, Belgium

${ }^{i}$ AZ Monica Euwfeestkliniek, Antwerpen, Belgium

${ }^{j}$ ZNA Middelheim, Antwerpen, Belgium

${ }^{\mathrm{k}} \mathrm{CHU}$ Sart Tilman, Liege, Belgium

Received 11 August 2011; received in revised form 22 September 2011; accepted 2 November 2011

KEYWORDS

Ulcerative Colitis;

Infliximab;

Calprotectin

\begin{abstract}
Aim: To evaluate the effect of infliximab induction therapy on calprotectin levels in patients with ulcerative colitis (UC).

Patients and Methods: In this prospective study 53 patients with active UC from 17 centers were treated with infliximab therapy $(5 \mathrm{mg} / \mathrm{kg}$ ) at baseline, week 2 , and week 6 . Faecal calprotectin was measured every week. Sigmoidoscopies were performed at baseline, week 6 and week 10 . Results: Median calprotectin levels decreased from 1260 (IQR 278.5- 3418 ) at baseline to 72.5 (IQR 18.5 - 463) at week $10(\mathrm{p}<0.001)$. After 10 weeks, infliximab therapy induced endoscopic remission and a decrease in calprotectin to $<50 \mathrm{mg} / \mathrm{kg}$ or at least a $80 \%$ decrease from baseline level in $58 \%$ of patients.
\end{abstract}

\footnotetext{
* Corresponding author.

E-mail address: martine.devos@ugent.be (M. De Vos).
} 
A significant and steep decrease of calprotectin levels was seen at week 2 for patients with an endoscopic remission at week 10 as compared to patients who did not show a remission. $(\mathrm{p}<0.001)$.

At week 10 an excellent correlation was found between endoscopic remission and clinical Mayo score reflected by an AUC of ROC analyses of 0.94 (0.87-1) and with calprotectin measurements (AUC $0.91(0.81-1))$ : all patients with calprotectin levels $<50 \mathrm{mg} / \mathrm{kg}$, and a normal clinical Mayo score $(=0)$ were in endoscopic remission.

Conclusions: Infliximab induces a fast and significant decrease of faecal calprotectin levels in anti-TNF naïve patients with ulcerative colitis predictive for remission of disease

(c) 2011 European Crohn's and Colitis Organisation. Published by Elsevier B.V. All rights reserved.

\section{Introduction}

Although the role of TNF-alpha in the pathogenesis of ulcerative colitis has been debated, Infliximab has been proven to be effective for the induction and maintenance of clinical remission and mucosal healing, resulting in a corticosteroidsparing effect. ${ }^{1,2}$ In most of the studies and in daily practice, the clinical Mayo score is used to monitor the disease activity of ulcerative colitis. It is a scoring system including stool frequency, rectal bleeding and physician's global assessment. Since one third of the points is attributed to "physician's global assessment", the Mayo score is partly subjective. Mucosal healing defined by the absence of lesions is more objective and the ultimate goal of therapy but necessitates an endoscopy. Non-invasive markers including acute phase reactants like $\mathrm{C}$-reactive protein (CRP) and hematological markers as erythrocyte sedimentation rate (ESR) or albuminemia are cheap, repeatable but are often normal in mild to moderate ulcerative colitis.

Calprotectin is a neutrophil derived $36 \mathrm{kDa}$ calcium and zinc binding protein which can be measured in the stools. It is a very sensitive marker for inflammation in the gastrointestinal tract. Detection of calprotectin levels in stools is easy and very safe: it requires only minimal quantity of faeces, it is stable for more than one week at room temperature, is resistant to degradation, can be analyzed by a simple ELISA test.

Its value as marker for endoscopic and histological activity has been tested previously ${ }^{3-5}$ and it was suggested that it could predict relapses in UC. ${ }^{6,7}$ Recently its use as predictor for response to first or second line medical therapy has been suggested ${ }^{8}$ but not confirmed in another study. ${ }^{9}$

The present study aimed at evaluating the evolution of calprotectin levels under infliximab induction therapy and its correlation with mucosal healing as compared to Mayo score.

\section{Patients and methods}

\subsection{Patients}

Anti-TNF naïve patients with active UC from 17 Belgian centers were treated with infliximab therapy $(5 \mathrm{mg} / \mathrm{kg})$ at baseline, week 2 , and week 6 . According to the Belgian reimbursement criteria, patients were eligible for treatment with infliximab if they had a combined Mayo score of $>/=6$ including an endoscopic subscore of $>/=2$ despite adequate treatment with aminosalicylates and corticosteroids /immunosuppressive therapy for at least 3 months. Patients were evaluated at baseline, week 2, week 6 and week 10 by clinical Mayo score, questionnaire and lab testing (including CRP, erythrocyte sedimentation rate, albumin, hemoglobin, platelet count). Faecal calprotectin was measured every week. Sigmoidoscopies were performed at baseline, week 6 and week 10 and graded from 0 to 3 according to the Mayo endoscopy subscore. Endoscopic remission was further defined by a score of 1 or less.

Remission per protocol was defined as endoscopic remission AND a normalization of calprotectin levels $(<50 \mathrm{mg} / \mathrm{kg})$ or at least a $80 \%$ decrease from baseline at week 10 .

Patients on corticosteroids had to be treated with stable doses at least 2 weeks before the start of the study and if possible steroids were tapered from week 2 at fixed schedule.

This study was approved by the local ethical committees and patients signed informed consent.

\subsection{Determination of faecal calprotectin}

Every week, patients provided a small (typically a teaspoon) stool sample $(1 \mathrm{~g})$ in a plastic Container. Samples were frozen at $-20{ }^{\circ} \mathrm{C}$ within $48 \mathrm{~h}$.

If patients provided a stool sample at the same time point as the endoscopy, the sample was delivered immediately prior to the start of the bowel cleansing.

Faecal samples were collected with a disposable, breakable inoculation loop and entered into a disposable screw cap tube. The faeces weight was measured and the loop handle broken off, leaving the loop and $4-6 \mathrm{~cm}$ of the handle inside the tube. Extraction solution containing urea and citrate was added. After $30 \mathrm{sec}$ agitation on a mixer followed by homogenization for $20 \mathrm{~min}$ at $1400 \mathrm{rpm}$ on a shaker, $1 \mathrm{ml}$ of the homogenate was transferred to an Eppendorf tube and centrifuged for $20 \mathrm{~min}$ at $10000 \times \mathrm{g}$. The supernatant $(0.5 \mathrm{ml})$ was collected and analyzed immediately or frozen at $-20{ }^{\circ} \mathrm{C}$ for later analysis.

Calprotectin levels were measured on the supernatant by enzyme linked immunosorbent assay (ELISA) (PhiCal ELISA kit, Eurospital Kit (SCIMEDX)) with standards and controls included and performed according to the manufacturer's instructions. Calprotectin levels were measured by a single central lab. Physicians were blinded for the results throughout the entire study.

\subsection{Statistical analysis}

For descriptive analysis, proportions, means and medians were calculated. Correlations between dichotomous outcome 
variables and continuous variables were evaluated by the calculation of area under the curve (AUC) of receiver operator curves $(\mathrm{ROC})$ with $95 \%$ confidence intervals $(\mathrm{Cl})$.

Analyses (intention to treat analysis =ITT) are based on the total number of patients including all available data and assuming missingness at random: for the calculation of proportions of remission, a regression based multiple imputation was performed including all recorded variables; for the evaluation of calprotectin titers over time, Linear mixed models analysis was used; the calculation of repeated measurements ROC analysis was performed after the calculation of predicted probabilities with GEE modeling. Apart a complete case analysis, further depicted with an asterisk ( $\left.{ }^{*}\right)$, was added based on the data from patients completing the study and patients who stopped the study due to inefficacy (considered as nonresponders). Based on estimates from the literature, the sample size of this study was calculated in order to have $>80 \%$ power to observe a significant decrease of calprotectin levels under effective UC therapy. All analysis were performed with PASW/SPSS18, Chicago, ill.

\section{Results}

Fifty-three patients were included. Mean disease duration was 6 years, and the mean age was 45 years, there were $48 \%$ female. Other baseline disease characteristics are mentioned in Table 1.

Table 1 Demographics and baseline characteristics.

\begin{tabular}{ll}
\hline Disease duration & $\begin{array}{l}\text { Mean 6 years } \\
(0-26)\end{array}$ \\
\hline Age & $\begin{array}{l}\text { Mean 45 years } \\
(18-78)\end{array}$ \\
Gender & $48 \%$ Female \\
Extent of disease (\% proctitis, \% left sided, & \\
\% extensive colitis) & \\
Initial extent & $30 \%-51 \%-19 \%$ \\
Maximal extent & $6 \%-61 \%-33 \%$ \\
Current extent & $13 \%-64 \%-23 \%$ \\
Smoking behavior (current, & $4 \%-43 \%-53 \%$ \\
$\quad$ ex-, non-smoker) & \\
Current Medication & \\
Mean number & $2.4(1-5)$ \\
Oral 5-ASA & $83 \%$ \\
Topical 5-ASA & $38 \%$ \\
Any ASA & $87 \%$ \\
Oral corticoids & $66 \%$ \\
Topical corticoids & $7.5 \%$ \\
Any corticoids & $68 \%$ \\
Methotrexate & $2 \%$ \\
Azathioprine & $47.2 \%$ \\
Clinical Mayo score (mean +range) & $6.3(0-9)$ \\
Endoscopic subscore & \\
- Mayo score 1 & $0 \%$ \\
- Mayo score 2 & $48 \%$ \\
- Mayo score 3 & $52 \%$ \\
\hline
\end{tabular}

\subsection{Attrition}

After 10 weeks, 2 patients stopped therapy due to inefficacy: corticosteroids were started in 1 patient; 1 patient underwent total colectomy. Three patients stopped due to safety reasons: 2 allergic reactions, 1 patient had a vertebral fracture.

Additionally, 12 patients had missing data, mostly due to withdrawal consent for endoscopy at the week 10 . Those patients were excluded from complete case analysis, further depicted with an asterisk ( $\left.{ }^{*}\right)$.

\subsection{Induction of remission under infliximab therapy}

After 10 weeks infliximab therapy induced endoscopic remission in $63 \%(\mathrm{Cl}: 47-78 \%)^{*}$ of patients. When all 53 patients were analyzed with multiple imputation, this overall remission rate decreased to $59 \%(\mathrm{Cl}: 46-72 \%)$.

Similarly, when the predefined definition of remission was used (Colonoscopy $</=$ grade 1 and, decrease of faecal calprotectin with $80 \%$ or faecal calprotectin levels $<50 \mathrm{mg} /$ $\mathrm{kg}), 58 \% *(\mathrm{Cl}: 43-73 \%)$ remission was obtained by complete case analysis and $54 \%(\mathrm{Cl}: 41-67 \%)$ by ITT analysis with multiple imputation. There was no difference in baseline Mayo score between responders and non responders in Mayo score (mean 6.5 vs. 6.4), Calprotectin levels (mean 1710 vs. $1680 \mathrm{mg} / \mathrm{kg}$ ) and endoscopic scores (median 2 vs. 2),

\subsection{Evolution of calprotectin levels under infliximab therapy}

Median calprotectin levels decreased from $1260 \mathrm{mg} / \mathrm{kg}$ (IQR 278-3418)* at baseline to $72.5 \mathrm{mg} / \mathrm{kg}(\mathrm{IQR} 18,5-463)^{*}$ at week 10. Further analysis showed that patients who went in endoscopic remission at week 10 showed a significant steep decrease between baseline and week $2(p<0.001)$ compared to patients who did not show a remission (Beta $=0.7,95 \% \mathrm{Cl} 0.27-1.12$ ). From week 3 on, this course was more flattened (Fig. 1). In this figure all available data from all patients were included. The line gives the result of the Linear mixed model analyses which was used to calculate the $p$-values.

The effect of Infliximab was similar in patients with and without concomitant use of corticosteroids (Fig. 2).

\subsection{Predictive value of week 2 calprotectin mea- surements to predict week 10 remission}

The steep decrease in calprotectin at week 2 predicted a remission at week 10 . However remission was not complete at week 2 since calprotectin level of $50 \mathrm{mg} / \mathrm{kg}$ or a decrease of at least $80 \%$ at week 2 predicted an endoscopic remission at week 10 with a specificity of $67 \%{ }^{*}$ and sensitivity of $54 \%{ }^{*}$. An additional effect was seen from second and to a less extent third infusion.

Absence of decrease in calprotectin levels at week 2 identified patients resistant to the treatment. 


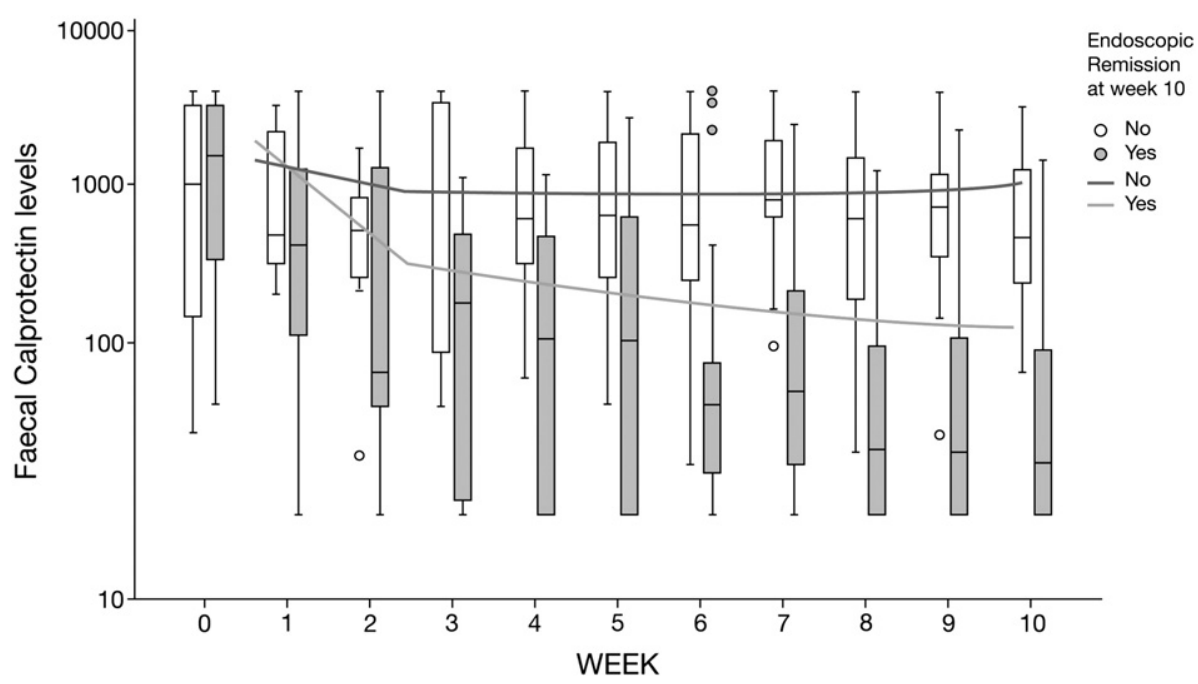

Figure 1 Evolution of calprotectin levels under infliximab therapy in function of the induction of endoscopic remission at week 10. Legend: Boxplots describe medians and interquartile ranges of the completersLines show the result of the mixed models analysis (ITT analysis).

\subsection{Correlation between calprotectin and other variables with endoscopic remission at the moment of endoscopy}

Clinical Mayo score $(=0)$ and calprotecin measurements ( $<50 \mathrm{mg} / \mathrm{kg}$ ) showed an excellent correlation with endoscopic remission, reflected by an AUC of ROC analyses of 0.94 (0.871) for Mayo score and $0.91(0.81-1)$ for calprotectin measurements at week $10^{*}$ : All patients $\left(63 \%^{*}\right)$ with a calprotectin level of $<50 \mathrm{mg} / \mathrm{kg}$, and all patients $\left(63 \%{ }^{*}\right)$ with a normal clinical Mayo score $(=0)$ were in endoscopic remission. This was significantly better than the IBDQ and serological parameters (Table 2). Similar results were obtained with an ITT repeated measures analysis taking into account all available endoscopic

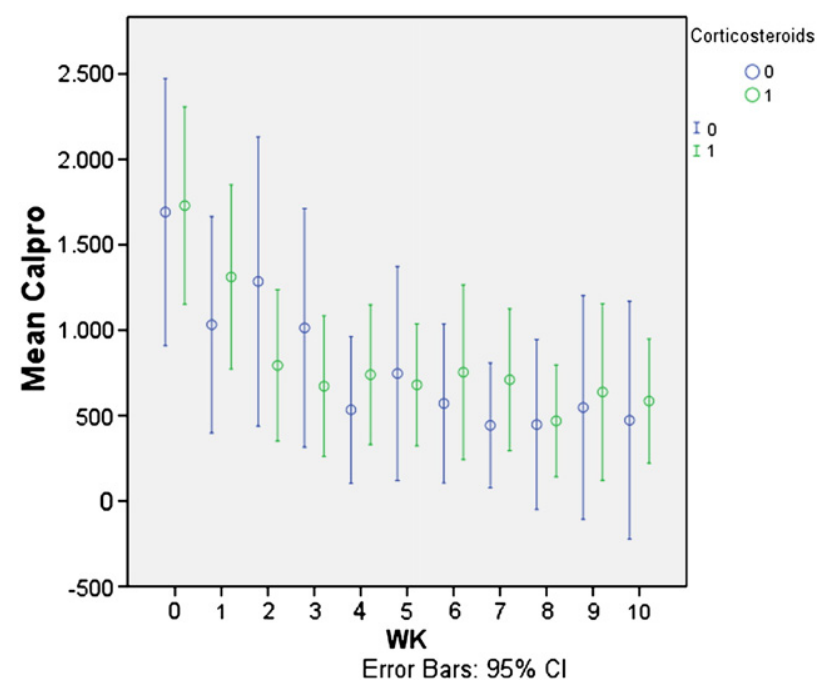

Figure 2 Evolution of calprotectin levels under infliximab therapy in function of the induction of endoscopic remission at week 10 and concomitant use of corticosteroids. Legend: Boxplots describe medians and interquartile ranges of the completers. Lines show the result of the mixed models analysis (ITT analysis). results : 0.97 (0.94-1) for the Mayo score and 0.85 (0.77-0.92) for the calprotectin measurements. Sensitivities and specificities of the different cut-offs of this analysis are provided in Table 2.

A similar correlation between calprotectin measurements and remission $(\mathrm{AUC}=0.89(0.82-0.97)$ ) was observed when remission was defined as a total sum score (Mayo and endoscopic score) less than 2 with no individual scores of $>1$ ).

\subsection{Overall correlation between calprotectin measurement and clinical Mayo scores}

The overall correlation and agreement between calprotectin measurements and the clinical mayo scores ranged from 0.3 to 0.45 , depending on the used statistical method (Spearsman's rho, Pearson $r$, Kappa), suggesting that both methods might be useful to combine in a classification model:

1. Combined measurement at the moment of colonoscopy :

A model combining both clinical Mayo score and calprotectin measurement as continuous variables, calculated by logistic

Table 2 Results of ROC analyses exploring the correlation between the different variables and endoscopic remission at week 10.

\begin{tabular}{llllll}
\hline & AUC & SE & P-value & $\begin{array}{l}\text { Lower } \\
\text { Bound }\end{array}$ & $\begin{array}{l}\text { Upper } \\
\text { Bound }\end{array}$ \\
\hline Mayo.10 (low) & 0.94 & 0.04 & 0.00 & 0.87 & 1.00 \\
Calprotectin (low) & 0.91 & 0.05 & 0.00 & 0.81 & 1.00 \\
IBDQ.10 (high) & 0.73 & 0.08 & 0.03 & 0.57 & 0.89 \\
Album.10 (high) & 0.67 & 0.09 & 0.09 & 0.50 & 0.85 \\
ESR.10 (low) & 0.65 & 0.10 & 0.15 & 0.45 & 0.85 \\
CRP.10 (low) & 0.64 & 0.10 & 0.18 & 0.45 & 0.83 \\
HB.10 (high) & 0.56 & 0.10 & 0.56 & 0.36 & 0.76 \\
PLT.10 (low) & 0.53 & 0.11 & 0.80 & 0.31 & 0.74 \\
\hline
\end{tabular}


regression analysis resulted in a small improvement in its correlation with endoscopic remission compared to the single measurements (AUC of ROC analysis 0.98 (.95-1.00) vs $0.97(0.94-1.00)$ for the Mayo score and $0.85(0.77-0.92)$ for the calprotectin measurements).

\section{Combined measurement at week 2 to predict remission at week 10}

A model combining both week 2 clinical Mayo score and week 2 calprotectin measurement as continuous variables, calculated by logistic regression analysis resulted in a small improvement in its correlation with endoscopic remission at week 10 compared to single measurements (AUC of ROC analysis $0.77(0.59-0.95)$ vs $0.73(0.53-0.93)$ for week 2 Mayo score and $0.59(0.38-0.79)$ for week 2 calprotectin measurements).

\section{Discussion}

In this prospective study infliximab induction therapy induced a complete endoscopic remission AND a decrease of calprotectin levels to less than $50 \mathrm{mg} / \mathrm{kg}$ or at least a $80 \%$ decrease from start levels in $58 \%$ of UC patients with active disease.

Although it is very well known in Crohn's disease that infliximab is associated with a rapid clinical effect and decrease in CRP levels already observed after 2 weeks, ${ }^{10}$ the effect in UC is less well- known. In ACT1 and 2 studies clinical improvement was observed as early as week $2 .{ }^{1}$ Similarly in our study we demonstrated a very fast effectiveness of treatment with a significant decrease of faecal calprotectin levels already observed 2 weeks after the first infusion .However an additional effect of second (and third) infusions was observed since value of $50 \mathrm{mg} / \mathrm{kg}$ or a decrease of at least $80 \%$ at week 2 predicted an endoscopic remission at week 10 with a specificity of $67 \%$ and a sensitivity of $54 \%$. Retrospective studies have been published in order to predict responders and included decrease in CRP level at week $2,{ }^{11}$ age at diagnosis or treatment or pANCA/ASCA status. ${ }^{12}$ In children with severe ulcerative colitis faecal concentrations of osteoprotegerin (decoy receptor for RANKL and regulator of apoptosis and cell survival) at day 3 have been identified as predictors for use of second line therapy. ${ }^{13}$ In adults with severe acute colitis calprotectin levels were higher in those requiring colectomy and were even useful to predict response to first and second line medication. ${ }^{8}$

Similar to ACT1 and ACT2 studies mucosal healing at 8 weeks was observed in $63 \%$ of patients. In our study mucosal healing (0-1) also was always associated with clinical remission and vice versa clinical Mayo score $=0$ was always associated with mucosal healing. In contrast mean partial Mayo score was still 2-3 in ACT1 and ACT2 studies correlating with a remission in only $28-39 \%$ of patients. We have no explanation for this difference but our finding was supported by a similar association with calprotectin levels of $50 \mathrm{mg} / \mathrm{kg}$ or less.

Blood laboratory measurements were less reliable to evaluate endoscopic remission.

In conclusion, infliximab induces a fast and sharp decrease in calprotectin levels already after the first infusion. The absence of this decrease seems to identify a group of non-responders . Calprotectin $<50 \mathrm{mg} / \mathrm{kg}$ is a very good predictor for mucosal healing and can possibly be used as additional for marker for deep remission.

\section{Conflict of interest}

There is no conflict of interest.

\section{Acknowledgement}

This study was supported by MSD without influence on the manuscript.

The Following centers of BIRD (Belgian IBD Research \& Development Group) participated in the current study

Dr. Amininejad , ULB-Erasme

Dr. Baert , H.Hartziekenhuis Roeselare-Menen

Dr. Caenepeel , ZOL, Genk

Dr. Coche, Clin St Pierre, Ottignies

Dr. Colard, Clin St Joseph, Luik

Dr. De Vos , UZ Gent

Dr. Dewit, UCL St Luc, Brussel

Dr. D'Haens , Imeldaziekenhuis, Bonheiden

Dr. Fontaine, Clin St Joseph, Luik

Dr. Franchimont , ULB-Erasme

Dr. Lambrecht, AZ Damiaan, Oostende

Dr. Lammens, Clin St Jean, Brussel

Dr. Louis, CHU Sart Tilman, Luik

Dr. Maisin , Clin de l'europe, Brussel

Dr. Mana , UZ Brussel

Dr. Moreels , UZ Antwerpen

Dr. Muls, CHU Saint Pierre, Brussel

Dr. Noman, UZ Leuven

Dr. Peeters , UZ Gent

Dr. Potvin , St Jozefkliniek, Bornem

Dr. Staessen , AZ Monica Eeuwfeestkliniek

Dr. Terriere , ZNA Middelheim, Antwerpen

Dr. Van Assche, UZ Leuven

Dr. Vander Cruyssen , UZ Gent

Dr. Van Outryve, AZ Monica Eeuwfeestkliniek

Dr. Vandervoort, OLV Ziekenhuis, Aalst

Dr. Vermeire , UZ Leuven

Author's contributions

All authors have made substantial contributions to all of the following: (1) the conception and design of the study, or acquisition of data, or analysis and interpretation of data, (2) drafting the article or revising it critically for important intellectual content, and (3) final approval of the version to be submitted.

MDV was principal investigator of the study and conducted the design.

BVC performed the statistical analysis. EL is the president of BIRD.

$M D V, E L$ and all other authors significantly contributed to the inclusion of patients.

\section{References}

1. Rutgeerts P, Sandborn WJ, Feagan BG, Reinisch W, Olson A, Johanns $J$, et al. Infliximab for induction and maintenance 
therapy for ulcerative colitis. N Engl J Med Dec 8, 2005;353(23): 2462-76 Erratum in: N Engl J Med. 2006 May 18;354(20):2200.

2. Barreiro-de Acosta M, Lorenzo A, Mera J, Dominguez-Muñoz JE. Mucosal healing and steroid sparing associated with infliximab for steroid-dependent ulcerative colitis. J Crohns Colitis Dec 2009;3(4):271-6.

3. Røseth AG, Aadland E, Jahnsen J, Raknerud N. Assessment of disease activity in ulcerative colitis by faecal calprotectin, a novel granulocyte marker protein. Digestion 1997;58(2): 176-80.

4. Costa F, Mumolo MG, Ceccarelli L, Bellini M, Romano MR, Sterpi $C$, et al. Calprotectin is a stronger predictive marker of relapse in ulcerative colitis than in Crohn's disease. Gut Mar 2005;54(3): 364-8.

5. Schoepfer AM, Trummler M, Seeholzer P, Criblez DH, Seibold F. Accuracy of four faecal assays in the diagnosis of colitis. Dis Colon Rectum Oct 2007;50(10):1697-706.

6. Gisbert JP, Bermejo F, Pérez-Calle JL, Taxonera C, Vera I, McNicholl AG, et al. Faecal calprotectin and lactoferrin for the prediction of inflammatory bowel relapse. Inflamm Bowel Dis Aug 2009;15(8):1190-8.

7. D'Incà R, Dal Pont E, Di Leo V, Benazzato L, Martinato M, Lamboglia $\mathrm{F}$, et al. Can calprotectin predict relapse risk in inflammatory bowel disease? Am J Gastroenterol Aug 2008;103(8): 2007-14.
8. Ho GT, Lee HM, Brydon G, Ting T, Hare N, Drummond H, et al. Faecal calprotectin predicts the clinical course of acute severe colitis. Am J Gastroenterol Mar 2009;104(3):673-8.

9. Turner D, Leach ST, Mack D, Uusoue K, McLernon R, Hyams J, et al. Faecal calprotectin, lactoferrin, M2-pyruvate kinase and S100A12 in severe ulcerative colitis: a prospective multicenter comparison of predicting outcomes and monitoring response. Gut Sep 2010;59(9):1207-12.

10. Targan SR, Hanauer SB, van Deventer SJ, Mayer L, Present DH, Braakman $\mathrm{T}$, et al. A short term study of chimeric monoclonal antibody CA2 to tumor necrosis factor alpha for Crohn's disease. N Engl J Med Oct 9, 1997;337(15):1029-35.

11. Jürgens $M$, Laubender RP, Hartl $F$, Weidinger $M$, Seiderer J, Wagner J, et al. Disease activity, ANCA and IL23R genotype status determine early response to infliximab in patients with ulcerative colitis. Am J Gastroenterol Aug 2010;105(8): 1811-9.

12. Ferrante M, Vermeire S, Katsanos KH, Noman M, Van Assche G, Schnitzler $F$, et al. Predictors of early response to infliximab in patients with ulcerative colitis. Inflamm Bowel Dis Feb 2007;13(2):123-8.

13. Sylvester FA, Turner D, Draghi II A, Uuosoe K, McLernon R, Koproske $\mathrm{K}$, et al. Faecal osteoprotegerin may guide the introduction of second-line therapy in hospitalized children with ulcerative colitis. Inflamm Bowel 2011;17(8):1726-30. 